INVESTIGACIÓN

\title{
LA GAMIFICACIÓN Y ARQUITECTURA FUNCIONAL: ESTRATEGIA PRÁCTICA EN EL PROCESO DE ENSEÑANZA/APRENDIZAJE USANDO LA TECNOLOGÍA
}

\section{GAMIFICATION AND FUNCTIONAL ARCHITECTURE: PRACTICAL STRATEGY IN THE TEACHING / LEARNING PROCESS USING TECHNOLOGY}

Ing. Leydi Liliana Hernandez Rojas ${ }^{a}$ Phd. Sir Alexci Suárez Castrillón ${ }^{\text {b }}$ MSc. Dewar RicoBautista $^{\mathrm{c}}$

a Ingeniera de Sistemas. Universidad Francisco de Paula Santander Ocaña - Semillero SIGLAS. Grupo de Ingeniería en Innovación, Tecnología y Emprendimiento - GRIITEM Ocaña, Colombia, 1lhernandezr@ufpso.edu.co

b Ingeniero de Sistemas, Doctor en Sistemas Inteligentes en la Ingeniería Universidad Francisco de Paula Santander Ocaña - Grupo de Investigación en Ciencia y Tecnología - GRUCITE. Ocaña, Colombia, sasuarezc@ufpso.edu.co

c Ingeniero de sistemas, Especialista en Telecomunicaciones, Magíster en Ciencias Computacionales, Estudiante Doctorado en Ingeniería. Universidad Francisco de Paula Santander Ocaña - Grupo de Ingeniería en Innovación, Tecnología y Emprendimiento - GRIITEM. Ocaña, Colombia, dwricob@ufpso.edu.co

Fecha de recepción: 01-04-2017

Fecha de aprobación: 22-06-2017

Resumen: Las Instituciones educativas actualmente no aplican una metodología que permita aprender de forma didáctica y creativa los temas en las aulas de clase, de lo cual el proceso de enseñanza se torna monótono y los estudiantes se desmotivan a la hora de aprender, llegando a la conclusión que los jóvenes no aprenden lo necesario y por lo tanto la indiferencia de los maestros por su débil formación didáctica y pedagógica hacen que haya deserción escolar. En este artículo se muestra la implementación de la Gamificación como metodología lúdica para llevar a cabo el proceso de enseñanza /aprendizaje en el uso de la tecnología. Esta metodología aprovecha todos los recursos posibles, combina tecnología, juego educativo y estrategias de refuerzo positivo, con el fin de motivar a los estudiantes en el aprendizaje.

Palabras clave: Educación; Gamificación; Innovación; Investigación; Proyección social.

Abstract: The educational institutions do not currently apply a methodology that allows learning in a didactic and creative way the topics in the classrooms, from which the teaching process 
becomes monotonous and the students are discouraged when it comes to learning, arriving at the conclusion that young people do not learn what is necessary and therefore the indifference of teachers due to their weak didactic and pedagogical training means that there is school desertion. This article shows the implementation of Gamification as a playful methodology to carry out the teaching / learning process in the use of technology. This methodology takes advantage of all possible resources, combines technology, educational games and positive reinforcement strategies, in order to motivate students in learning.

Keywords: Gamification; Innovation; Investigation; Social projection.

\section{INTRODUCCIÓN}

El juego tiene un papel fundamental en la vida de los niños y jóvenes. A través de él se divierten, crean, imaginan, se desarrollan tanto social como personalmente y además, también aprenden. Por lo tanto, la implementación de la metodología de la Gamificación en las Instituciones Educativas, es una estrategia pedagógica y didáctica que ayude a mejorar el aprendizaje de los estudiantes.

A lo largo de los años la forma de vida, los intereses y la educación cambian, por lo que debe haber una innovación en el proceso de enseñanza/aprendizaje, de tal manera que las metodologías empleadas se adapten a estos cambios y motiven e interesen al estudiante. En Educación se tiende a trabajar con métodos tradicionales, como la exposición, la memorización, la indagación, etc., aunque actualmente se emplean nuevas metodologías para el desarrollo del proceso de enseñanza/aprendizaje del estudiante. Por lo tanto es conveniente, como se ha mencionado anteriormente, innovar metodológicamente.

Nadie aprende si no le mueve una razón, por ello se debe motivar al estudiante a fin de mejorar su rendimiento académico y favorecer de forma progresiva el aprendizaje autónomo. Es esencial potenciar el interés, la responsabilidad y el deseo de esforzarse en el logro del trabajo bien realizado, Pero no solo basta con que el estudiante juegue. Imma Marín, Asesora pedagógica de la Fundación Crecer Jugando (2014), defiende que "no tiene sentido hablar de construir el aprendizaje a través del juego si no juegan las dos partes (profesores y alumnos)", es decir, que el profesor también ha de implicarse en el juego. Todos hemos aprendido jugando y siendo alumnos hemos deseado que el profesor nos sorprenda con algo nuevo que nos motive y nos haga partícipes del proceso enseñanza/aprendizaje (Marín, 2010).

Por lo tanto, el empleo de una metodología que tenga como base componentes de juego tales como recompensas, puntos, niveles, etc., puede dar lugar a un proceso de enseñanza/aprendizaje enriquecedor y motivador para el estudiante, lo que se convierte en el objeto de estudio del presente proyecto. Todos estos motivos son los que guían a este trabajo a la implementación del uso del juego en el aula, concretamente la técnica de Gamificación. Los juegos influyen de manera considerable en los niños y jóvenes, sobre todo, aquellos que premian y refuerzan positivamente.

\section{MARCO TEÓRICO}


En la década de los 80, comienza a hacerse popular el término TIC, muy común hoy en día, y que años atrás ya había surgido en algunos grupos particulares de intelectuales como pequeñas sociedades de informática. Esto refleja como en las diferentes comunidades a nivel mundial, en países en vía de desarrollo y quienes aún no estaban encaminados en este proceso, comienzan a apostar por un cambio desde una labor sistemática productiva industrializada, donde los nuevos eventos de las tecnologías de la información y comunicación (TIC) permite desempeñar un papel esencial, ya que debido a estas tecnologías se da paso al mejoramiento continuo de algunos sectores y de la actividad industrial.

EL origen de lo que hoy se conoce sobre el concepto de las TIC se da como resultado de la convergencia tecnológica de la electrónica, el software y la infraestructura de las telecomunicaciones. La unión de estas tecnologías da paso al nacimiento del proceso de la información, donde las comunicaciones dan por hecho el surgimiento de nuevos horizontes $y$ paradigmas. Las tecnologías de la información y las comunicaciones comienzan a masificarse a finales de la década de los 70 y principios de $\operatorname{los} 80$, a partir de ese momento se comienza ver en estas tecnologías, el gran potencial que puede ofrecer en los sectores educativos. A finales de los años 80, viendo las ventajas y lo que representa la inclusión de estas tecnologías, se emprende en los países desarrollados la elaboración de una serie de iniciativas para involucrar a las TIC en la formación de los estudiantes en los diferentes centros educativos.

Hoy en día se puede apreciar que las TIC son un pilar básico en las sociedades y fundamental en el proceso de desarrollo para el crecimiento profesional y personal en los jóvenes, la incorporación de estas tecnologías en la educación favorecen el aprendizaje ya que facilitan los medios en los que se logra el desarrollo de los conocimientos.

Bien se sabe que las TIC están produciendo grandes cambios en la forma de cómo ha evolucionado la adquisición del conocimiento informático en las personas, poblaciones, empresas, gobiernos y academias. Se resalta la gran participación que entra a formar las TIC en los sectores de la educación razón por la cual ha sido indispensable analizar y reestructurar los procesos de enseñanzaaprendizaje de los centros de educación media y de las universidades con el objetivo de alcanzar las metas propuestas por el gobierno. Se observa también como el país ha adoptado políticas sobre las TIC en los sectores productivos y educativos de Colombia, obteniendo una serie de resultados favorables.

Actualmente la incorporación de las TIC en las actividades académicas ha dado la posibilidad para desarrollar habilidades y destrezas en las prácticas docentes en la Educación Superior. Teniendo en cuenta las estrategias de enseñanza-aprendizaje, es importante tener presente que la mayoría de los estudiantes de pregrado son personas nacidas en los años 90, que ya contaban con acceso a los medios digitales, correo electrónico y contenidos multimedia, entre otros.

Este acercamiento temprano a las tecnologías, una constante comunicación y navegación en la redes con acceso casi ilimitado a la información que se puede encontrar en ella, facilita la inclusión de ciertas estrategias tecnológicas en los procesos de aprendizaje, generando un trasformación cultural en la construcción 
de conocimiento donde de igual forma se produce un rol nuevo en el docente e inventando nuevos desafíos en el sector educativo. (Cristancho Prada \& Valencia Plata, 2013)

\section{METODOLOGIA}

En el desarrollo del proyecto se implementó una arquitectura funcional, (ver figura 1), aplicada a los estudiantes para motivarlos a la hora de aprender, utilizando metodologías diferentes a la pedagogía tradicional.

\section{Arquitectura Funcional}

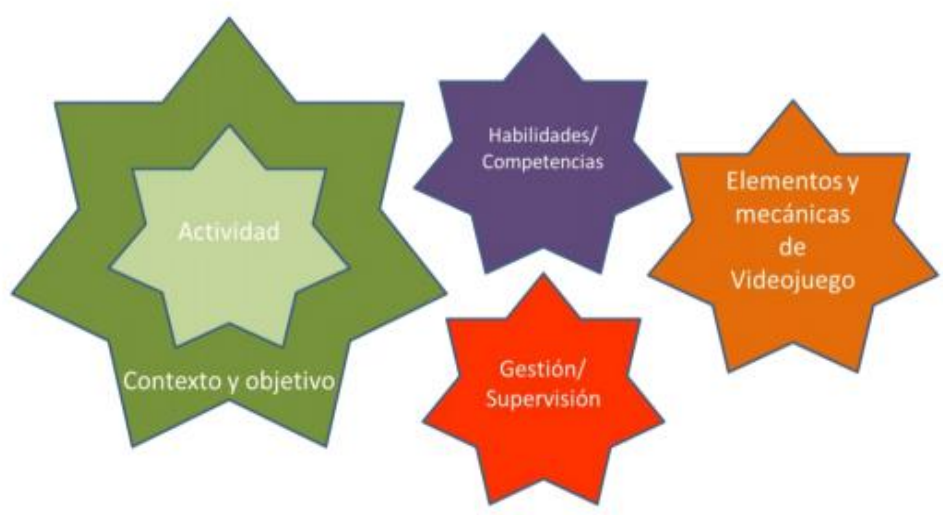

Figura 1. Arquitectura funcional. Fuente: Autor

\subsection{La actividad}

Representa una serie de acciones dentro de la lógica de aprendizaje motivada en el cerebro, para conseguir estimular la resolución de problemas necesarios para alcanzar un objetivo deseado y que una vez resuelta liberará los mecanismos químicos del cerebro asociado con su funcionamiento básico.

Estas actividades no deben ser definidas de forma estándar o de forma genérica independientemente de la audiencia o de los usuarios a los que van destinados, pero teniendo en cuenta las habilidades que poseen las personas/sistemas, y en el caso de la Gamificación del aula, de los alumnos y de sus edades. El no tenerlo en cuenta puede limitar o anular el éxito del proceso de Gamificación (Contreras, 2016).

\subsection{Contexto y objetivo}

El aula, se integra dentro de un contexto más amplio que de alguna forma condiciona las posibilidades de las técnicas que van a utilizar, porque de alguna forma, cuanto más amplio y transversal sea el proceso más éxito va a tener. El contexto organizacional de la institución influirá de forma directa sobre la libertad y la capacidad del profesor de poder gamificar su AULA.

\subsection{Habilidades y competencias}

Cada estudiante, tiene un conjunto de competencias y habilidades que son propias de su persona, y han sido desarrolladas de forma continua a lo largo de los años, no por estar en la misma clase, se tienen esas mismas 
características, y tratar de homogenizar esta concepción puede provocar la desestabilización del proceso de Gamificación porque las diferentes percepciones y respuestas que generará en alumnos con diferentes habilidades. De la misma forma, como las habilidades sociales son muy fuertes en estos jóvenes, los resultados negativos pueden ser rápidamente compartidos por el grupo $\mathrm{y}$ llevar el proceso a ser un completo fiasco.

Es necesario calibrar correctamente las actividades y sus intensidades para conseguir la inmersión completa y homogénea en el proceso, dado que, de esta forma, la visión será compartida por todos y no aparecerán los mensajes disonantes dentro del grupo (Contreras, 2016).

\subsection{Gestión y supervisión}

En cualquier sistema o transformación de un sistema, es necesario contar con una fuerte posición de liderazgo y de especialidad, que puede conducir con seguridad y autoridad el proceso dentro de todos los contextos definidos en el punto anterior.

La dirección tiene que ser una parte integral de todo el proceso, para referenciar los resultados, las recompensas, los enfoques, y también para poder respaldar el proceso siempre que sea necesario, a través de la potencial reacción de los elementos exógenos de la organización, y con los stakeholders del sistema, como podrían ser los padres y las autoridades educativas (Contreras, 2016).

\subsection{Elementos y mecánicas del Juego}

El modelo de juego realmente funciona porque consigue motivar a los alumnos, desarrollando un mayor compromiso de las personas, e incentivando el ánimo de superación. Se utilizan una serie de técnicas mecánicas y dinámicas extrapoladas de los juegos. (Gaitán, 2013).

- Técnicas Mecánicas: Es la forma de recompensar al usuario en función de los objetivos alcanzados, en estos encontramos acumulación de puntos, escalado de niveles, obtención de premios y clasificadores

- Técnicas Dinámicas: Hacen referencia a la motivación del propio usuario para jugar y seguir adelante en la consecución de sus objetivos, entre estos encontramos recompensas, estatus, logros y competición.

\section{MATERIALES}

Los dispositivos seleccionados como herramientas de apoyo fueron Arduino Uno y Raspberry Pi 2, puesto que son los más idóneos para enseñar temáticas relacionadas con dicha carrera, como son lenguajes de programación, sistemas operativos, electrónica y redes de computadores;

\subsection{Arduino}

Arduino es una plataforma de electrónica abierta para la creación de prototipos basada en software y hardware libre, flexible y fácil de usar. Se creó para artistas, diseñadores, aficionados y cualquier interesado en crear entornos $\mathrm{u}$ objetos interactivos. Arduino puede tomar información del entorno a través de sus pines de entrada, para esto puede ser usada una gran gama de sensores y puede afectar otros componentes que lo rodean, controlando luces, motores y otros actuadores. 
El microcontrolador en la placa Arduino se programa mediante el lenguaje de programación Arduino (basado en Wiring: entorno de programación de entradas/salidas de código abierto) y el entorno de desarrollo Arduino (basado en Processing: lenguaje de programación orientado a la creación visual y plataforma de aplicaciones computacionales altamente interactivas). Los proyectos hechos con Arduino pueden ejecutarse sin necesidad de conectarlo a un ordenador, si bien tienen la posibilidad de hacerlo y comunicar con diferentes tipos de software (p.ej. Flash, Processing, MaxMSP). Las placas pueden ser hechas a mano o comprarse montadas de fábrica; el software puede ser descargado de forma gratuita. Los ficheros de diseño de referencia (CAD) están disponibles bajo una licencia abierta, así pues, eres libre de adaptarlos a tus necesidades (Monk, 2012).

El Arduino Uno es una placa electrónica basada en el ATmega328P. Cuenta con 14 pines digitales de entrada / salida (de los cuales 6 se pueden utilizar como salidas PWM), 6 entradas analógicas, un cristal de cuarzo de 16 $\mathrm{MHz}$, una conexión USB, un conector de alimentación, una cabecera ICSP y un botón de reinicio. Contiene todo lo necesario para apoyar el microcontrolador; simplemente conectarlo a un ordenador con un cable USB o el poder con un adaptador de CA o la batería a CC para empezar, (ver figura 2).

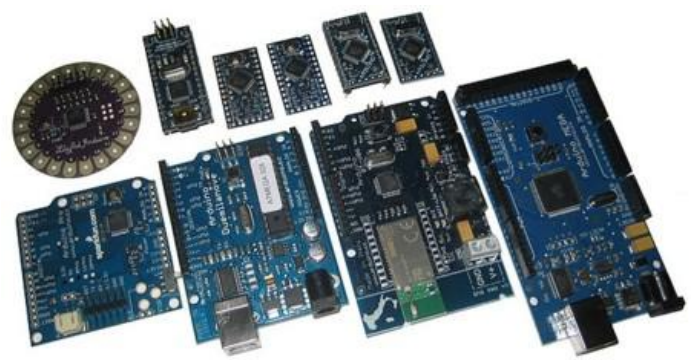

Figura 2. Arduino. Fuente: Arduino.cc

\subsection{Raspberry PI 2}

Es un ordenador de placa reducida de bajo coste desarrollado por la Fundación Raspberry Pi en Reino Unido, con el objetivo de estimular la enseñanza de ciencias de la computación en las escuelas. Esta placa no incluye una unidad de almacenamiento como un disco duro o disco de estado sólido, pues hace uso de una tarjeta SD para almacenamiento permanente. Cuenta con una memoria RAM de $1 \mathrm{~Gb}$, un procesador QuadCore a $900 \mathrm{MHz}$, cuatro puertos USB, un puerto Ethernet. Para su uso, la fundación y comunidad Raspberry Pi han desarrollado una cantidad de sistemas operativos de arquitectura ARM para estos dispositivos, siendo en su mayoría basados en GNU/Linux. Se promueve con la Raspberry el aprendizaje de varios lenguajes de programación como Python, Tiny Basic, C, Perl y Ruby, (ver figura 3).

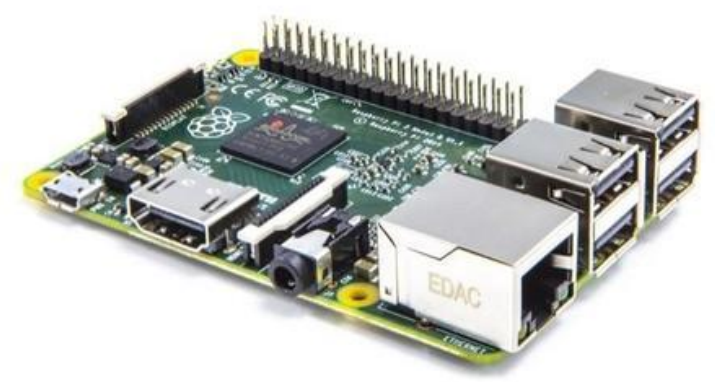

Figura 3. Raspberry Pi 2. Fuente: Raspberypi.org

\section{ESTRATEGIA}

En este proyecto se diseñaron todos los contenidos, utilizando videos y presentaciones alusivos al tema, (ver figura 4).

También se diseñaron los ejercicios y actividades de las plataformas Arduino y Raspberry; el cual está conformada por sopa de letras, descubrir parejas, relacionar elementos, mapas, crucigramas, 
ahorcados, ordenar palabras, rompecabezas y quiz, (ver figura 5).

Durante el proceso los resultados se obtuvieron a través de las calificaciones y asistencias al curso de Gamificación, donde a cada estudiante se le acumulo las notas realizadas de los ejercicios y actividades, (ver Figura 6).

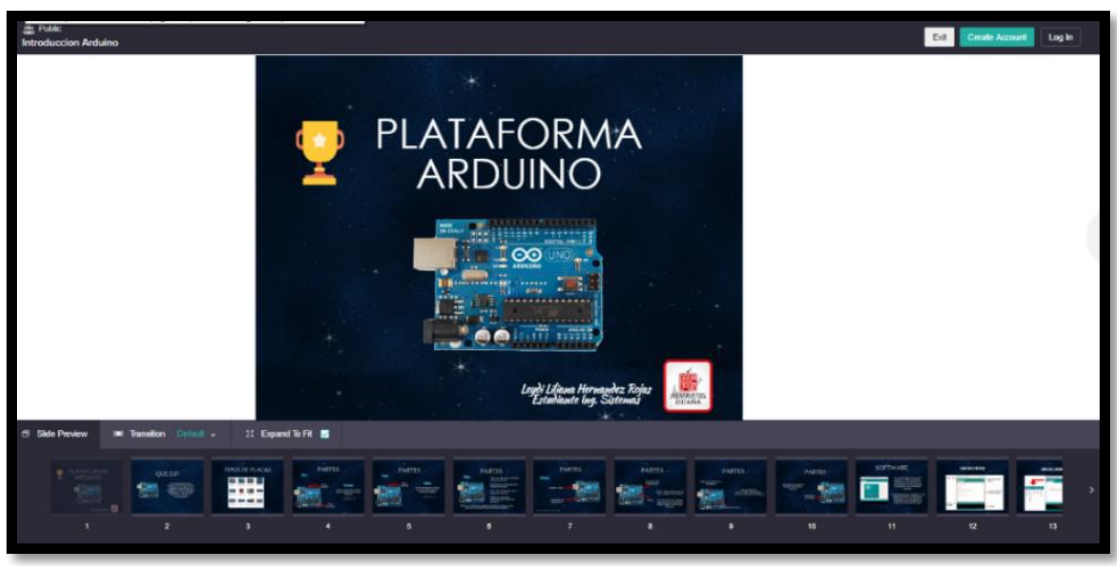

Figura 4. Presentación en Piktochart de la Introducción a la Plataforma de Arduino. Fuente: Autor

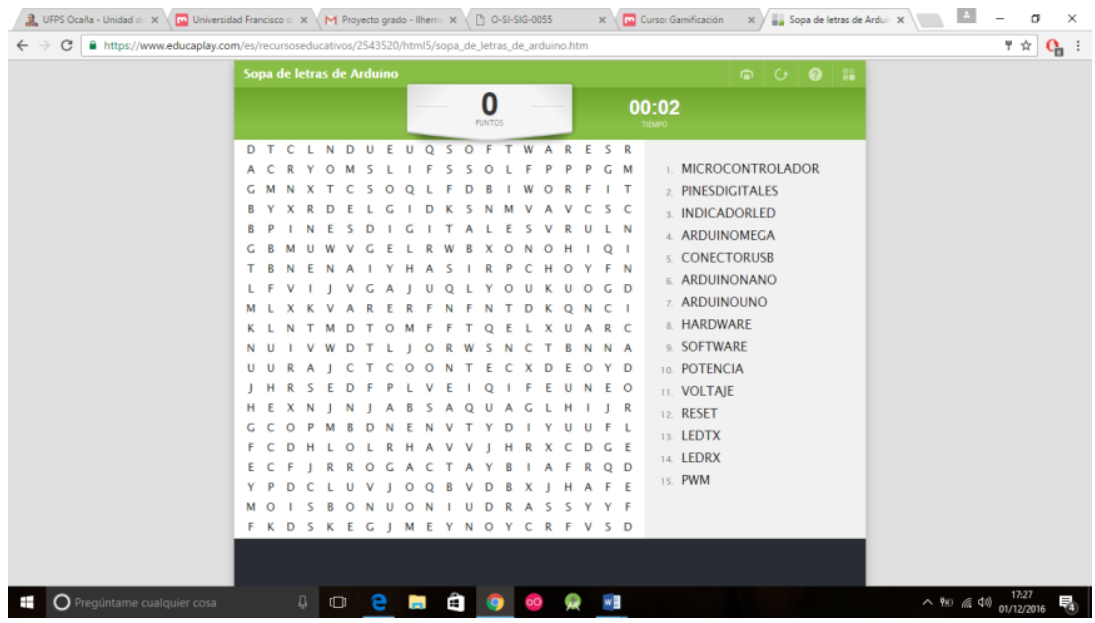

Figura 5. Actividad: Sopa de Letras de Arduino en Educaplay. Fuente: Autor

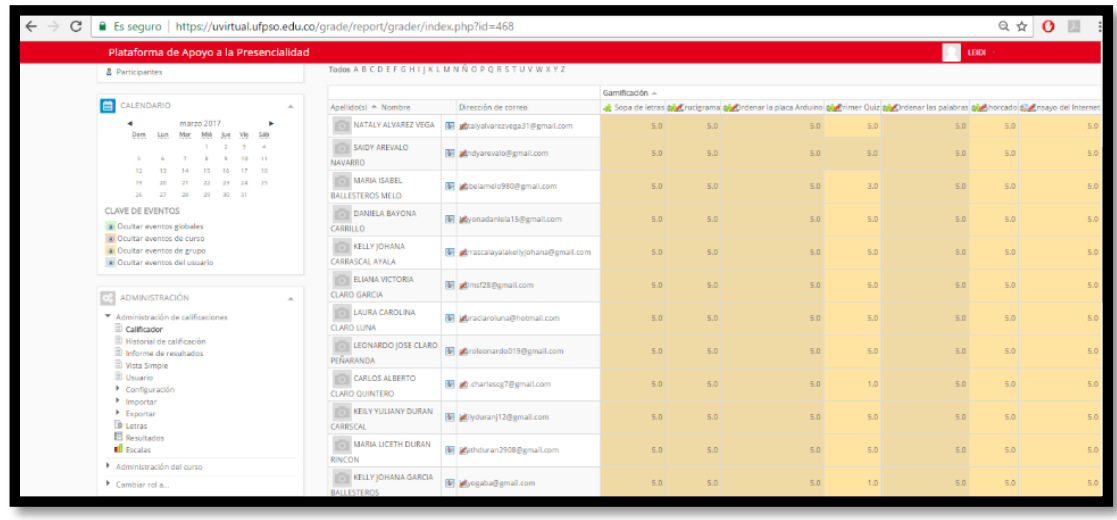

Figura 6. Calificaciones de los Estudiantes del Curso de Gamificación. Fuente: Unidad de Educación Virtual UFPSO

Por último, se llevó a cabo el desarrollo de todas las capacitaciones, prácticas, ejercicios y actividades del módulo de Gamificación con los estudiantes del 
grado Decimo del colegio Fray José María Arévalo del Municipio de la Playa de Belén, (ver figura 7).

Durante todo el desarrollo del curso de Gamificación, se fueron acumulando puntos positivos, se tuvo en cuenta la asistencia de los estudiantes y también las calificaciones de todos los ejercicios, actividades y prácticas desarrolladas en clase, con el fin de dar premiación a los mejores estudiantes del curso de Gamificación.

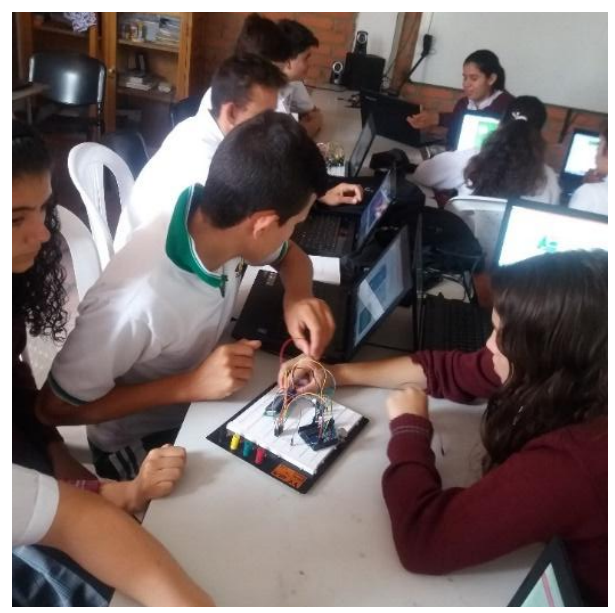

Figura 7. Evidencia Fotográfica del Desarrollo de la Práctica: Pantalla LCD con Arduino. Fuente: Autor

Para el desarrollo de esta actividad se realizó la clausura del curso de Gamificación el último día de clases de los estudiantes del colegio Fray José María Arévalo, donde se obtuvieron varias premiaciones como son: el mejor estudiante del curso, el mejor grupo del curso y mejor estudiante de competencia, (ver figura 8).

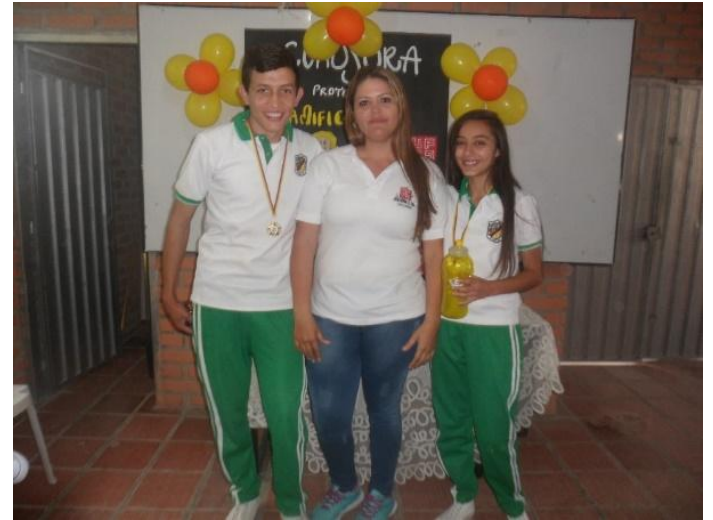

Figura 8. Premiación. Fuente: Autor

Durante la clausura se realizaron una serie de juegos incentivando al estudiante a participar de manera activa en cada una de las actividades programadas, (ver figura 9).

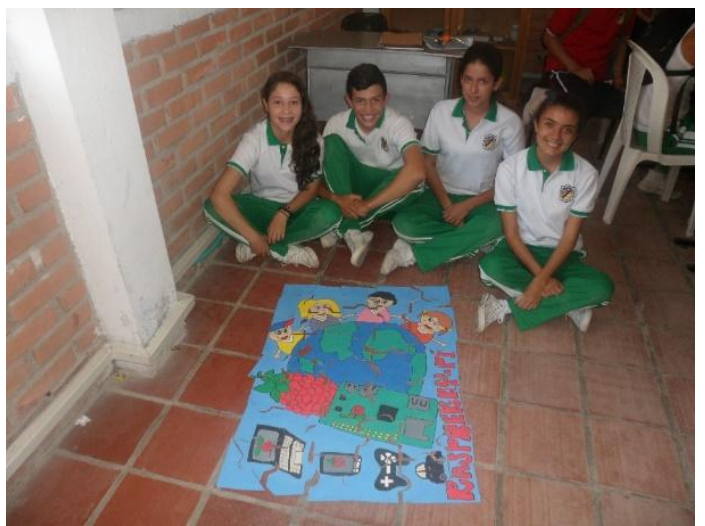

Figura 9. Evidencia Fotográfica del Mejor Grupo Ganador del Curso. Fuente: Autor

\section{RESULTADOS}

Se desarrolló un módulo virtual, donde cada estudiante tenía acceso a la plataforma, con el fin de interactuar directamente con ella, (ver tablas 1 y 2 ).

Tabla 1 Descripción de las Técnicas Mecánicas utilizadas en el Curso

TECNICA

ACUMULACION PUNTOS

\section{DESCRIPCION DEL RESULTADO}

DE Se obtuvo a través de las puntuales asistencias de los estudiantes al curso y las dinámicas realizadas del tema del día. 
ESCALADO DE NIVELES

CLASIFICADORES

REGALOS
Se obtuvo a través de las competencias en el desarrollo de los ejercicios y actividades (el primero que iba ganando subía de nivel)

Se obtuvo mediante la elaboración de las prácticas de la Plataformas Arduino y Raspberry (se detectaba los más agiles)

Se premiaron a los estudiantes con mejor nivel, puntal asistencia, desarrollo de todas las prácticas del curso y los grupos ganadores durante todas las clases.

Fuente: Autor

Tabla 2 Descripción de las Técnicas Dinámicas Obtenidas en el Curso

TENICAS

RECOMPENSAS

ESTATUS

LOGRO

COMPETICION

\section{DESCRIPCION DEL RESULTADO}

Se obtuvieron a través de las dinámicas realizadas del tema del día.

Se obtuvo a través de los ejercicios y actividades desarrollados, también de la elaboración de cada una de las prácticas de las Plataformas Arduino y Raspberry.

Se obtuvo de cada una de los ejercicios y prácticas desarrollados en clase (motivación elevada del estudiante).

Se obtuvo durante el desarrollo de todas las clases, donde cada estudiante quería ser el mejor y ocupar el primer puesto; además que todo lo realizado en el curso generaba notas para las materias de Física e Informática y eso era de gran motivación para competir durante cada clase.
Fuente: Autor

\section{FINANCIAMIENTO}

La Universidad Francisco de Paula Santander Ocaña (UFPSO), mediante la División de Investigación y Extensión (DIE) se vincula a docentes, administrativos y estudiantes para que participen en la ejecución y desarrollo de proyectos de investigación. Esta ponencia muestra resultados de 2 proyectos inscritos, avalados y financiados en dicha dependencia:

- "Seguridad en redes inalámbricas", propuesto a través del Grupo de Investigación en Ingenierías Aplicadas (INGAP).

- "Inseguridad en las redes sociales e internet: prioridad en las escuelas de la provincia Ocaña”, propuesto a través del Semillero de Investigación GNU/Linux And Security (SIGLAS) del Grupo de Investigación en Ingenierías Aplicadas (INGAP).

De igual forma muestra resultados del proyecto de grado inscrito y avalado a través del comité curricular del plan de estudios de ingeniería de sistemas:

- "IMPLEMENTACION DE LA GAMIFICACION EN EL PROCESO DE ENSEÑANZA/APRENDIZAJE EN EL USO DE LA TECNOLOGIA", propuesto a través del Semillero de Investigación GNU/Linux And Security 
(SIGLAS) del Grupo de Investigación en Ingenierías Aplicadas (INGAP).

\section{CONCLUSIONES.}

Se logró demostrar que la metodología de la Gamificación es indispensable para el proceso de enseñanza/aprendizaje; ya que el estudiante al interactuar con la tecnología y aplicando herramientas educativas, hace que su desempeño educativo sea de mejor agrado y por lo tanto se motive a estudiar con más dedicación.

También se realizó control de calificación y asistencias de los estudiantes al módulo de Gamificación; de lo cual fue un estímulo positivo ya que cada uno se motivó a esforzarse y a estar preparado a la hora de llegar a la clase y por lo tanto la ausencia de estudiantes al curso era mínima.

Al momento de aplicar esta metodología los estudiantes lograron mejores resultados tanto en la elaboración como desarrollo de todas las prácticas y actividades propuestas en el módulo implementado; con este módulo el estudiante participo de manera individual logrando acumular puntos $\mathrm{y}$ alcanzo un nivel superior, con el objetivo de una premiación al final del proyecto; esta metodología hizo que cada estudiante se motivara a estudiar y a reforzar los temas, con el fin de llegar ser el mejor estudiante del curso.

De los jóvenes encuestados un alto porcentaje afirmaron que ha tenido experiencia realizando alguna práctica de electrónica y programación en su institución como por ejemplo armado de circuitos utilizando protoboard. Este resultado reafirmó que la selección de la institución ha sido la mejor ya que su afinidad por la tecnología ha desarrollado competencias que facilitaron el desarrollo del proyecto.
Los procesos de enseñanza y de aprendizaje han mejorado gracias a la incorporación de las TIC en la educación (Carneiro, Toscano, \& Diaz, 2014). Sin embargo, en la aplicación del proyecto se puede observar que no es suficiente para el programa de ingeniería de sistemas con la publicidad realizada hasta el momento, también es importante abordar el tema de las competencias digitales de los profesores, así como es necesario avanzar en la incorporación de las nuevas tecnologías en la familia $y$ hogares como apoyo $y$ acompañamiento para reducir la brecha digital incluyendo temas tales como la seguridad de la información, videojuegos, sistemas operativos, redes de datos en los jóvenes de $10^{\circ}$ y $11^{\circ}$ secundaria.

Entre los jóvenes encuestados, un alto porcentaje afirmó que le interesaría aplicar la programación en aplicaciones móviles, videojuegos, software educativo y a páginas web. Esto refleja el impacto que ha tenido el Ministerio de Tecnologías de la Información y las Comunicaciones (MinTIC) y su plan Vive Digital para promover y potenciar la creación de negocios a partir del uso de las TIC, poniendo especial interés en el desarrollo de aplicaciones móviles, software y contenidos (Ministerio TIC, 2011).

\section{REFERENCIAS}

Adams Becker, S., Johnson, L., Gago, D., Garcia, D., \& Martín, S. (2013). Perspectivas Tecnológicas > Educación Superior en América Latina 2013-2018. Texas: The New Media Consortium.

Adell, J., \& Castañeda, L. (2012). Tecnologías emergentes ¿Pedagogías emergentes? Tendencias emergentes en educación con TIC, 13-32. 
Aisyah, A. R. (2013). The Development of Working Design through Characterized Technology Pedagogy and Content Knowledge in the Elementary Schools' Instructional. Procedia - Social and Behavioral Sciences, 1016-1024.

Arancibia, V., Herrera , P., \& Strasser, K. (2008). Manual de Psicología Educacional. Santiago de Chile: Ediciones Universidad Católica de Chile.

Bandera Carvajal, N. S. (2012). Implementación de estrategias de promoción y divulgación para la Facultad de Ingeniería Informática de la Universidad Pontificia Bolivariana Seccional Bucaramanga. Bucaramanga.

Barletta, N., Toloza, H., del Villar, L., Rodríguez, A., Bovea, V., \& Moreno, F. (2013). Enseñanza y aprendizaje de la lectura y escritura: una confabulación en el contexto oficial. Lenguaje, 133-168.

Bonilla Cárdenas , S. (2011). Estructura económica y desesmpleo en Colombia: un análisis VEC. Sociedad y Economía, 99-124. Obtenido de http://socioeconomia.univalle.edu.co /revista

Bulut, B., Çakmak, Z., \& Kara, C. (2013). Global Citizenship in Technology Age from the Perspective of Social Sciences. Procedia - Social and Behavioral Sciences, 442-448.

Caicedo Tamayo, A. M., \& Rojas Ospina, T. (2014). Creencias, conocimientos y usos de las TIC de los profesores universitarios. Educación $y$ Educadores, 517-533.

Castrillón López, L. A. (2014). Universidad y desarrollo humano. Escritos, 1317.
Chiappe, A., \& Cuesta, J. C. (2013). Fortalecimiento de las habilidades emocionales de los educadores: interacción en los ambientes virtuales. Educación y Educadores, 503-524.

Coll, C. (2013). El currículo escolar en el marco de la nueva ecología del aprendizaje. Aula de Innovación Educativa, 31-36.

Correa Ramírez, J. D., \& Montoya Arbelaez, J. A. (2013). El valor del capital humano: una aproximación desde el enfoque del ingreso para Colombia, 2001-2009. Cuadernos de economía, 32-60.

Cortizo Pérez, J. C., Carrero García, F., Monsalve Piqueras, B., Velasco Collado, A., Diaz del Dedo, L. I., \& Pérez Martín, J. (2011). Gamificación y Docencia: Lo que la Universidad tiene que aprender de los Videojuegos. VIII Jornadas Internacionales de Innovación Universitaria Retos y oportunidades del desarrollo de los nuevos títulos en educación superior.

Cristancho Prada, A. M., \& Valencia Plata, G. F. (2013). Integración de las TIC en los procesos de enseñanza aprendizaje en la Universidad Piloto de Colombia. Pre Til(29), 31-42.

Danaj, L., Dumi, A., Zejneli, I., \& Çelo, E. (2013). The Improvement of Capacity of Administrative and Local Government Using the Strategic Planning of E-Learning in Albania. Procedia - Social and Behavioral Sciences, 1-9.

DANE y la Agenda de Conectividad. (2003). Modelo de la Medición de las Tecnologías de la Información y las Comunicaciones - TIC. Resumen Ejecutivo. Recuperado el 24 de 04 de 2015, de 
http://www.dane.gov.co/files/investi gaciones/tics/tics.pdf

Díaz, F., \& Hernández , G. (2002). Estrategias de enseñanza para la promoción de aprendizajes significativos. En Estrategias docentes para un aprendizaje significativo, una interpretación constructivista (2da ed., págs. 69112). México: McGraw Hill.

Enríquez, S. C. (2012). Luego de las TIC, las TAC. II Jornadas Nacionales de TIC e Innovación en el Aula.

Escorcia Oyola, L., \& Jaimes de Triviño, C. (2015). Tendencias de uso de las TIC en el contexto escolar a partir de las experiencias de los docentes. Educación y Educadores, 137-152.

Ferreiro, R. (2006). Mas allá de la teoría: El Aprendizaje Cooperativo: El Constructivismo Social. Revista Magister. Obtenido de http://www.redtalento.com/Articulos /WEBSITE\%20Revista\%20Magister $\% 20$ Articulo\%206.pdf

Gaitán, V. (Octubre de 2013). Gamificación: el aprendizaje divertido. Obtenido de http://www.educativa.com/blogarticulos/gamificacion-elaprendizaje-divertido/

Gallego, G. (2014). Universidades y desarrollo económico local. Perfil de coyuntura económica, 9-14.

Gómez Pinto, L. R. (2014). La política en educación comom determinante de desarrollo económico: El contexto histórico sobre la tensión de modelos económicos. Vniversitas, 121-152. doi:doi:10.11144/Javeriana.VJ128.pe cd

González González, C. S. (2015). Técnicas de gamificación aplicadas en la docencia de Ingeniería Informática. Obtenido de ReVision, 8(1).: http://www.aenui.net/ojs/index.php?j ournal $=$ revision $\&$ page $=$ article $\&$ op
$=$ viewArticle \&path $\% 5 B \% 5 D=152 \&$ path $\% 5 B \% 5 D=290$

Gordillo, M. (2009). Educación, ciencia, tecnología y sociedad. Madrid: Centro de Altos Estudios Universitarios de la OEI. Recuperado el 23 de 04 de 2015, de http://www.oei.es/DOCUMENTO3c aeu.pdf

Grupo de análisis, monitoreo y gestión de la Información. (2015). Boletín Educación Superior en Cifras. Bogotá: Ministerio de Educación .

Hurtado, J. A., Collazos, C. A., Cruz, S. T., \& Rojas, O. E. (2012). Child Programming: Una Estrategia de Aprendizaje y Construcción de Software Basada en la Lúdica, la Colaboración y la Agilidad. Revista Universitaria en Telecomunicaciones, Informática y Control, 9-14.

Igado, M. F. (2013). La'gamificación'como motivación en los entornos virtuales de aprendizaje. Obtenido de In II congreso internacional Eduación Mediática y Competencia Digital. Ludoteracy, creación colectiva y aprendizaje (pp. 228-250).: https://scholar.google.es/scholar?q=e nse \%C3\%B1anza+y+aprendizaje+en + gamificacion\&btnG $=\& h l=e s \& a s \_s$ $\mathrm{dt}=0 \% 2 \mathrm{C} 5$

Imma, M. (2010). "Jugar, una necesidad y un derecho". ALOMA revista de la facultad de la psicologia , num. 25.

Instituto de Tecnologías Educativas. (2011). Iniciativa 1:1. España: Ministerio de Educación.

KAPP, K. M. (2012). The Gamification of Learning.

Marín, I., \& Hierro, E. (2013). Gamificación El poder del juego en la gestión empresarial y en la conexión con los clientes. Barcelona: Urano / Empresa activa. 
Martínez Cuellar, C. (2013). El efecto de la desigualdad y el acceso al crédito sobre la acumulación de capital humano. Ensayos sobre política económica, 18-34.

Melchor Ferrer, E. (2012). Gamificación y e-Learning: un ejemplo con el juego del pasapalabra. EFQUEL, 137-144.

Mellado, V. (2014). La formación y el desarrollo profesional del profesorado de ciencias experimentales. Tecné, Espisteme y Didaxis TED, 7-10.

Migración Colombia. (2014). Boletín Anual de Estadísticas 2014. Bogotá: Ministerio de Relaciones Exteriores.

Ministerio de las TIC. (5 de Marzo de 2014). Noticias. Obtenido de MINTIC: http://www.mintic.gov.co/portal/604/ w3-article-1629.html

Ministerio TIC. (Febrero de 2011). Plan Vive Digital 2010 - 2014. Recuperado el 1 de Diciembre de 2015 , de http://www.mintic.gov.co/images/M S_VIVE_DIGITAL/archivos/Vivo_ Vive_Digital.pdf

Monk, S. (2012). 30 Proyectos con Arduino. España: Estribor.

Muñoz Rojas, H. A., \& Núñez Valero, J. G. (2010). Las políticas públicas educativas y las tecnologías de la información y la comunicación (Tic) en colombia: una caracterización desde 1991 al 2008. Magistro, 79-89.

Netmarketshare. (2005). Netmarketshare. Recuperado el 1 de Diciembre de 2015 , http://www.netmarketshare.com/

Nocua, A. P. (2015). Talento Digital: esencia transofrmadora del país. RevistaCIO@Gov,12-13. Obtenido de

http://issuu.com/mintic_col/docs/_ci o_40gov_no.3_abril-junio_2015_
Padiha, M., \& Aguirre, S. (2011). La integración de las TIC en la escuela. Indicadores cualitativos $y$ metodología de investigación. Argentina: OEI Fundación Telefónica.

Páramo, P., \& Hederich, C. (2014). Educación basada en la evidencia. Revista Colombiana de Educación, 13-16.

Planells de la Maza, A. J. (2013). La emergencia de los Game Studies como. Historia y Comunicación Social, 519-528. doi:http://dx.doi.org/10.5209/rev_HI CS.2013.v18.43985

Puentes Ossa, E., \& Clavijo Ballesteros, J. C. (2012). GNU/Linux Sistema Operativo y Servicios.

Pyster, A., Adcock, R., Ardis, M., Cloutier, R., Henry, D., Laird, L., . . W Wade, J. (2015). Exploring the Relationship between Systems Engineering and Software Engineering. Procedia Computer Science, 708-717.

REDIS. (2010). I Encuentro Nacional de Ingeniería de Sistemas. Hacia una prospectiva de la profesión en Colombia. Retos de la Ingeniería de Sistemas al 2015. Paipa: REDIS.

Reig, D., \& Vílchez, L. F. (2013). Los jóvenes en la era de la hiperconectividad: tendencias, claves y miradas. Madrid: Fundación Telefónica, Fundación Encuentro.

Román, M., Cardemil, C., \& Carrasco, A. (2011). Enfoque y metodología para evaluar la calidad del proceso pedagógico que Incorpora TIC en el aula. (R. I. (RINACE), Ed.) Revista Iberoamericana de Evaluación Educativa, 4(2), 8-35. Obtenido de http://hdl.handle.net/10486/661645

Romero Trenas, F. (2009). Aprendizaje Significativo y Constructivismo. Temas para la Educación. Obtenido 
de

http://www.feandalucia.ccoo.es/docu /p5sd4981.pdf

Şenyuva, E., \& Kaya, H. (2014). Procedia Social and Behavioral Sciences. ERPA International Congress on Education, ERPA Congress 2014, 68 June 2014, Istanbul, Turkey, 386392.

Serna, A., \& Serna , E. (2013). ¿Está en crisis la ingeniería en el mundo? Una revisión de literatura. Revista Facultad de Ingeniería Universidad de Antioquia, 199-208.

Serna, E., \& Serna, A. (2015). Crisis de la Ingeniería en Colombia. Ingeniería y Competitividad, 17(1), 63-74.

Serrano Gonzáles - Tejero, J. M., \& Pons Parra, R. M. (2011). El constructivismo hoy: enfoques constructivistas en educación. Revista Electrónica de Investigación Educativa, 1-27.

Sosa, E., \& Godoy, D. (2014). Internet del futuro. Desafíos y perspectivas. Revista de Ciencia y Tecnología, 16(21), 40-46. Recuperado el 30 de Noviembre de 2015, de http://www.scielo.org.ar/pdf/recyt/n2 1/n21a07.pdf

Storm, S. (2015). Crisis and recovery in the German economy: The real lessons. Structural Change and Economic Dynamics, 11-24. doi:http://dx.doi.org/10.1016/j.struec o.2015.01.001.

Tanenbaum, A. (2012). Redes de Computadores. México: Pearson.

TIOBE Company. (2000). TIOBE Software $B V$. Recuperado el 01 de Diciembre de 2015, de http://www.tiobe.com/index.php/con tent/paperinfo/tpci/index.html

Trejos Buriticá, O. I. (2014). Relaciones de aprendizaje significativo entre dos paradigmas de programación a partir de dos lenguajes de programación. Tecnura, 91-102.

Ulloa, G. (2010). ¿Qué pasa con la Ingeniería en Colombia? Ingeniería \& Sociedad, 1-4.

Universia Colombia. (26 de Febrero de 2014). Ciencia y Nuevas tecnologías. Obtenido de Universia: http://noticias.universia.net.co/cienci a-nntt/noticia/2014/02/26/1084885/infor me-consumo-internet-adolescentescolombia.html

Urh, M., Vukovic, G., Jereb, E., \& Pintar, R. (2015). The model for introduction of gamification into e-learning in higher education. Procedia - Social and Behavioral Sciences, 388-397.

Yigit, T., Koyun, A., Yuksel, A. S., \& Cankaya, I. A. (2014). Evaluation of Blended Learning Approach in Computer Engineering Education. Procedia - Social and Behavioral Sciences, 807-812. 